\title{
Identification of Nonlinear Predictor and Simulator Models of a Cement Rotary Kiln by Locally Linear Neuro-Fuzzy Technique
}

\author{
Masoud Sadeghian and Alireza Fatehi
}

\begin{abstract}
One of the most important parts of a cement factory is the cement rotary kiln which plays a key role in quality and quantity of produced cement. In this part, the physical exertion and bilateral movement of air and materials, together with chemical reactions take place. Thus, this system has immensely complex and nonlinear dynamic equations. These equations have not worked out yet. Only in exceptional case; however, a large number of the involved parameters were crossed out and an approximation model was presented instead. This issue caused many problems for designing a cement rotary kiln controller. In this paper, we presented nonlinear predictor and simulator models for a real cement rotary kiln by using nonlinear identification technique on the Locally Linear NeuroFuzzy (LLNF) model. For the first time, a simulator model as well as a predictor one with a precise fifteen minute prediction horizon for a cement rotary kiln is presented. These models are trained by LOLIMOT algorithm which is an incremental tree-structure algorithm. At the end, the characteristics of these models are expressed. Furthermore, we presented the pros and cons of these models. The data collected from White Saveh Cement Company is used for modeling.
\end{abstract}

Keywords - Cement rotary kiln, nonlinear identification, Locally Linear Neuro-Fuzzy model.

\section{INTRODUCTION}

$\mathrm{C}$ EMENT production is a complex process which is composed of a series of activities requiring considerable technological support [1]. A cement rotary kiln is a nonlinear and distributed parameter system that demonstrates timevarying nonlinear behavior due to the chemical reactions. The basic process in a cement production plant is baking the raw material mixed in a rotary kiln. Its task is to take an appropriate mixture of input material and to gradually burn and bake it to produce clinker [2].

Cement rotary kiln is the most important part of a cement factory whose outcome is cement clinker. A rotary kiln is a cylinder with a length of around 70 meters and a diameter of around 5 meters in a factory with a capacity of producing

Masoud Sadeghian is with the Mechatronics Department, School of Science and Engineering, Sharif University of Technology International Campus, Kish Island, Iran,(phone:+98-913-113-4382; e-mail: m_sadeghian@kish.sharif.edu).

Alireza Fatehi is with Advanced Process Automation \& Control (APAC), Mechatronics Department, School of Electrical engineering of K.N. Toosi University of Technology, Tehran, Iran (e-mail: fatehi@kntu.ac.ir). about 2000 tons of clinker in a day. The kiln is rotated by a powerful electrical motor. The temperature in the hottest point in the kiln is up to $1400^{\circ} \mathrm{C}$.

Control of a cement kiln is complicated by the fact that several factors such as nonlinearity, dynamism, huge size and MIMO with a gross interference are involved. Having different purposes, such as control, prediction and simulation, most researchers attempt to identify this complicated system and try to design an accurate model expressing the intricate and nonlinear operational procedure of the kilns.

Recently, some attempts have been made to develop computational fluid dynamics based models (CFD) to simulate either calciner [3] or kiln [4-5].Besides, the technique of thermo chemical process simulation has been used to compile a model that enables the chemical reactions to be calculated in various process stages (preheater, calcinatory, rotary kiln including the transition zone and sintering zone and cooler) [6]. Some attempts have also been made to develop reaction engineering models for kiln [7]. The numerical experiments using the computational model, could also predict the influence of kiln-operating parameters in it. Although that some significant results have been obtained in the past years, it is evident that there are many uncertainties involved in the models. None of the above theoretical models for kilns are sufficient as predictive tools for rotary kiln operation. So, more efforts should be made to model the kiln that could demonstrate its behavior. It is expected that the model will be a useful tool to industry in many situations.

On the other hand, intelligent and automatic systems have an important role in both the academia and industrial applications. Artificial neural networks, fuzzy systems [8] and neuro-fuzzy systems [9-10], show some main fields of computational intelligence, which have many applications ranging from prediction to control and system identification [11].These techniques provide powerful tools for non parametric analysis of nonlinear systems model-free processing and control of uncertain systems and plants.

One of the most important parts of modeling a system is to select the proper model structure. The other parts are dependent to selection of a suitable model structure; this issue is so important that imperfection in this section may lead to some sever problems in other parts and all the costs and attempts made to overcome the problem would be in vain. By 
using the previous result of identification of cement rotary kiln project [12], the linear identification techniques could not present a suitable model for cement rotary kiln; and consequently using a nonlinear identification technique is more suitable for this propose [13-14].

In this work, to continue the previous effort to design nonlinear models, an attempt is being made to improve the models and make more accurate ones. In this manner, a fourteen-week operation of the kiln was considered and the new collected data was pre-processed. Then a proper model structure is selected by analyzing data and after that a model is produced. Two LLNF models are designed for rotary kiln. The first model is prediction type which has been used before, regarding only one output, a prediction horizon of roughly seven minutes and the data collected based on two-week operation of the kiln [13, 15]; however, in this paper the prediction model presents based on all four outputs, a prediction horizon of fifteen minutes with higher accuracy and a fourteen-week operation of the kiln. This model can be useful in fault detection subject. The second model is a simulator type. This model is presented for the first time in this paper. This model is useful to study kiln behavior in different conditions and designing the model-based controller.

The paper is organized as follows: In the next Section, the selection procedure of inputs and outputs will be discussed. Afterwards, the pre-processing stages of the data for acquiring appropriate identification data will be presented. Next, we will calculate input channels delay estimation. Then, we will briefly discuss LLNF model and the training procedure of the parameters of this network based on Locally Linear Model Tree (LOLIMOT) technique and the results of predictor and simulator models for the kiln are presented and analyzed. At the end, the advantages and disadvantages of each of the above said models and conclusion will be discussed.

\section{SELECT INPUT AND OUTPUT VARIABLES}

As mentioned in the introduction, the cement rotary kiln has a complicated system due to the physical operation such as martial feed rate, fuel and air flow, kiln speed as well as chemical reactions. Operators usually can apply some inputs. These commands for the kiln model can be construed as the model inputs. Furthermore, main sensors in this section could be selected as output variables in the model. To identify the rotary kiln of the White Cement Saveh Company, the input and output variables, based on process engineering and operators experience, are shown in tables 1 and 2.

TABLE I INPUT VARIABLES IN KILN

\begin{tabular}{|l|c|}
\hline \multicolumn{1}{|c|}{ Input variables } & Variable name \\
\hline Material Feed Rate & Mat \\
\hline Fuel Feed Rate & Fuel \\
\hline Kiln Speed & Ks \\
\hline I.D Fan Speed & Fan \\
\hline Secondary Air Pressure & Ap \\
\hline
\end{tabular}

By selecting these inputs and outputs, it is possible to make a model that can show the real kiln behavior. Identification of the cement rotary kiln with these input and output variables is identification of a system with 5 inputs and 4 outputs. However, identification of a MIMO system is a hard task; Moreover, the results are not accurate. Therefore, we study this system as 4 MISO plant and try to identify these four subsystems [16].

\section{DATA USED IN MODELING}

Three important factors in modeling by system identification methods are:

1) Collection of correct and valid data

2) Selection of a suitable and useful model

3) A powerful method to adjust model based on information.

One of the most important assumptions to get valid information from an input and an output is that the changes happened in the output are affected by the system input and not disturbance or noise. As we collected input and output data during operation of the kiln to identify system behavior, we should check the changes in outputs that are more influenced by inputs. Its interference from disturbance inputs is lower, the validity of data would be better. Therefore, data mining is one of the most important and difficult steps in identification in order to extract valid data from the available data.

\section{A. Collection and selection of proper data}

To identify a model for plant, the collected data for identification should be reliable enough in order to illustrate various dynamics of the system. To do this, various signals such as PRBS and Chirp have been used to simulate and obtain dynamics of the system in numerous books and references. However, our plant is a real industrial factory and because of safety and operation limitations, it is impossible to give various signals with different frequencies to the system. Therefore, we must use the available data from normal operation. On the other words, identification is passive type. To be able to make an accurate and full identification, fourteen-week data are collected from cement factory. Having considered and analyzed the fourteen-week data, we made an effort to eliminate constant, repetitive and faulty operational points. The reason why we eliminated the constant and repetitive points is that in this condition the variables are affected just by the small noise and disturbance. In practice the generated dynamics by these data are not the main dynamics. On the other hand, if the volume of the used data for making a model is large in one point, this point gets more weight and increases error in other operating points. It shows that for modeling the cement rotary kiln, it is impossible to 
use raw data that are collected from factory. Consequently, it is necessary to use a big size of data, and then, analyzing various operation points in order to provide reliable data that could express system behavior.

There are several reasons why we could not use the original data collected from the kiln for identification. These reasons include high frequency noise or offset existing in the input and output data. Therefore, the raw data is not applied directly to indentify the system. To overcome some of these problems, we should try to use some pre-processing methods presented in identification references [17] which will be discussed in the next part.

\section{B. Sampling Frequency}

In computer controlled systems, sampling of continuous signals leads to annihilation of some information. Therefore, it is essential to select a syntactic sampling frequency which does not interfere with the control system. Although high frequency sampling seems better for control propose, it may cause some problems in system identification. Three methods are proposed for sampling for the purpose of system identification [17].
1) Smallest Time constant
$\mathrm{T}_{\mathrm{s}}=\tau_{\min } / 3$
2) Band width
$\mathrm{f}_{\mathrm{s}}=10 \mathrm{f}_{0}$
3) Settling Time
$\mathrm{T}_{\mathrm{s}}=\mathrm{T}_{\mathrm{st}} / 20$ to $\mathrm{T}_{\mathrm{st}} / 100$

In this paper, in order to obtain sampling frequency, all inputs and outputs signal spectra are drawn and we obtain frequency range of each one of them. Studying the power spectrum of the output signals shows that the smallest time constant of the system is 3 minutes. Based on the equation 1 the rate of sampling was assumed to be 60 seconds.

\section{Peak Shaving}

Initially, we have to consider and observe the input and output data, in order to realize the dynamism that may occur in the system, to a certain degree. Peak shaving and smoothing intense changes in data are very important. These sudden changes may occur due to the operation of sensors or data acquisition cards. They cause some numerical problems in measuring and recording variables as well. This may happen because the sensor is turned off suddenly for instance, when it needs to substitution or repaired. Recall that the used data in this project have been collected when the factory was operating. These sudden changes have a lot of energy in high frequency rang that degrades estimation of the parameters of the models or validity rate [12]. To solve this problem, the data are passed through a proper filter that can eliminate noise signal from original signal. But all signals have various signal spectra and to design band pass filter, we face with many problems. For example, if we increase the width of band pass filter, it will cause some quantities of noise signal to remain on the original signal; and if we decrease the width of band pass filter, it will cause some input signals to perish and sometimes change the input signals shape. You can see these situations in figures 1 and 2. Figure 1 shows the result of data that passes through a high frequency band pass filter; consequently the noise signal remains on the original signal.
Figure 2 shows the effect of adjustment of band pass filter to a low frequency band pass filter; although it can eliminate noise signal but it changes original signal shape. To solve this problem we need a filter with swift descent characteristic. Therefore, the Chebyshev filter is selected and all signals are passed through this filter. But after observing the result we understood because of its frequency characteristics, it causes fluctuation in settling status. Thus, we select a first order Butterworth-low-pass filter that has a proper band pass. By selecting $0.0015 \mathrm{HZ}$ band width, it is possible to eliminate noise and disturbing signals from original signal so that the input signals are not affected. Figure 3 shows the result of using this filter.

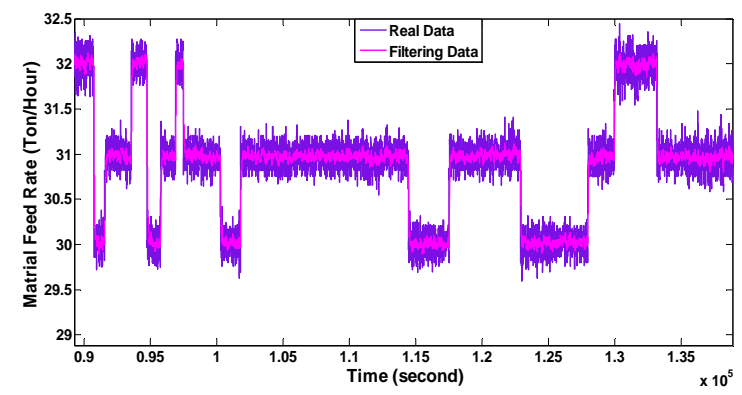

Fig. 1 The effect of high band pass filter on data

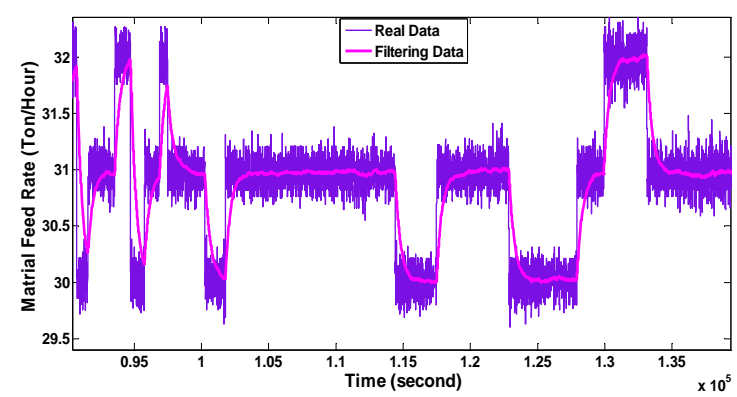

Fig. 2 The effect of using low band pass filter on data

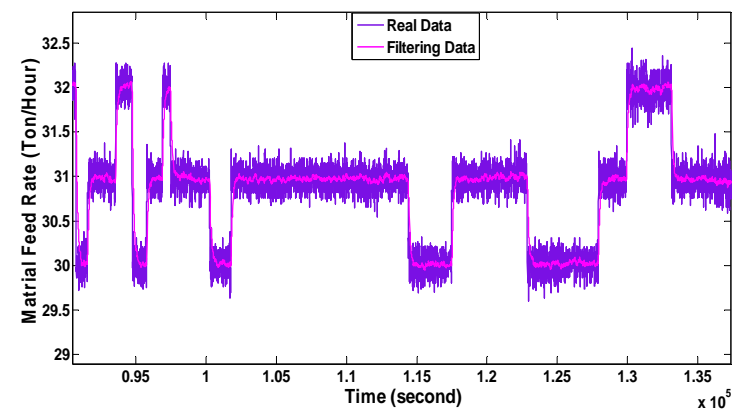

Fig. 3 The effect of using Butterworth filter on data

\section{Normalizing Data}

In this part, we have tried to normalized data. The reason why we want to normalize data is that the inputs and outputs data have different ranges that cause error in data quantization. This increases the quantization error during identification phase, and consequently the plant not identified well. Therefore, to identify the model, it is better to normalize all the data Figure 4 shows both the original and the normalizing of one of the input signals. 


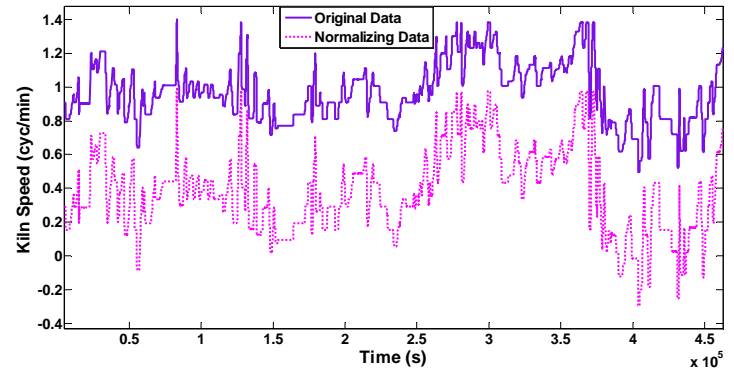

Fig. 4 Original data and Normalized data for Kiln speed (Ks)

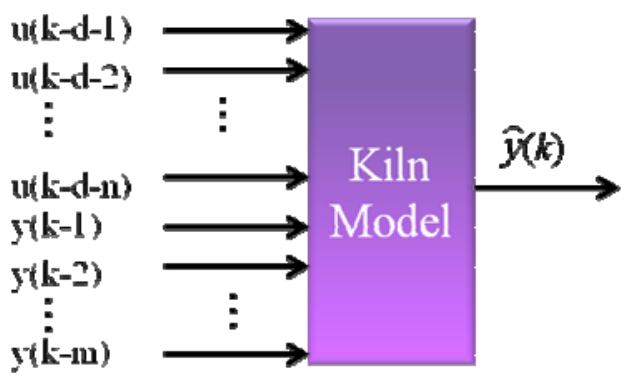

Fig. 5 Structure of prediction model

\section{E. Input Channels Delay Estimation}

In the identification of the process, the first parameter that should be determined is pure delay estimation. While this characteristic is correctly estimated from the process data, the identification of process simplified and gets suitable order. On the other hand, estimation of delay simultaneous to other parameters of model causes an increase in the computational efforts. This problem in the kiln model identification where each MISO model has five different inputs and a vast content of data is exposed more difficulties in system identification. In order to obtain inputs delay estimation, various methods such as step response test, cross correlation, analysis and mutual information analysis are applied. In this paper, we used the Lipschitz method that has been presented by Makarmi et al [18]. The results are shown in table 3.

TABLE III INPUTS AND OUTPUTS DELAYS
\begin{tabular}{|c|c|c|c|c|}
\hline & \multicolumn{5}{c|}{ Delays (min) } \\
\hline Variables & Pre & BE & CO & Ka \\
\hline Mat & 30 & 18 & 15 & 10 \\
\hline Fuel & 10 & 4 & 5 & 25 \\
\hline Ks & 40 & 36 & 5 & 0 \\
\hline Fan & 5 & 0 & 0 & 10 \\
\hline Ap & 5 & 0 & 3 & 30 \\
\hline
\end{tabular}

\section{IDENTIFICATION TECHNIQUE}

As mentioned in the previous sections, linear methods do not present a suitable model for rotary kiln. Using LLNF network for kiln identification is more proper. Thus, in this section, the kiln is identified by two prediction and simulation techniques.

\section{A. Prediction Technique}

Figure 5 shows the structure of model predictor used for identification of the kiln. Two different groups of inputs $u(k-$ $\mathrm{d}-\mathrm{i})$ and the previous real process outputs $\mathrm{y}(\mathrm{k}-\mathrm{i})$ are given to the model where the future output $\hat{y}(k)$ will be predicted. Inputs to the model are the delayed inputs of the plant and previous samples of the output of the plant.

\section{B. Simulation Technique}

Figure 6 shows a simulation method for a plant. As you see, in this technique only the plant inputs are given to the model in addition to the previous outputs of the model and the outputs which are obtained from pervious steps of the model but not from outputs of the real plant.

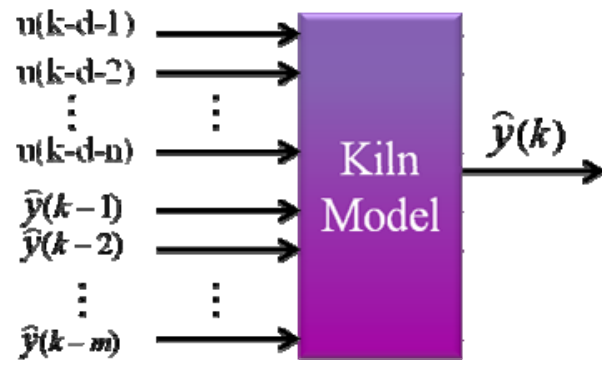

Fig. 6 Structure of simulation model

\section{Locally Linear Neuro-Fuzzy Network}

In the preceding section, we estimated the input channel delays of the kiln. Knowing these parameters, the search space for the identification shrinks and it's easier to do the rest of the job, i.e. determining the suitable number of dynamics on each input and the output, and approximating the best function which represents the behavior of the kiln as well. We use Locally Linear Neuro-Fuzzy (LLNF) network to identify the kiln and the LOLIMOT algorithm to find the best structure and parameters of the network. The most important reasons why LLNF network is selected is its:

1) High accuracy

2) Robustness

3) Computational efficiency and

4) Smooth switch for multiple models.

In the following LLNF networks and the LOLIMOT algorithm is reviewed briefly. Then the result of applying them on kiln data is represented.

The network structure of LLNF is depicted in Fig. 7. Each neuron realizes a Local Linear Model (LLM) and an associated validity function that determines the region of validity of the LLM. The network output is calculated as a weighted sum of the outputs of the local linear models, where the validity function is interpreted as the operating point dependent weighting factors. The validity functions are typically chosen as normalized Gaussians.

The local linear modeling approach is based on a dividedand-conquer strategy. A complex rotary kiln model divided 
into a number of smaller and thus simpler sub-problems, which are solved independently by identifying simple linear models $[11,19]$. The most important factor for the success of this model by a locally linear model method is the division strategy for the original complex problem. This will be done by an algorithm named LOLIMOT (Locally Linear Model Tree). LOLIMOT is an incremental tree construction algorithm that partitions the input space by axis-orthogonal splits [10]. In each iteration, a new rule or local linear model is added to the model and the validity functions that correspond to the actual partitioning of the input space are computed, and the corresponding rule consequence are optimized by a local weighted least squares technique.

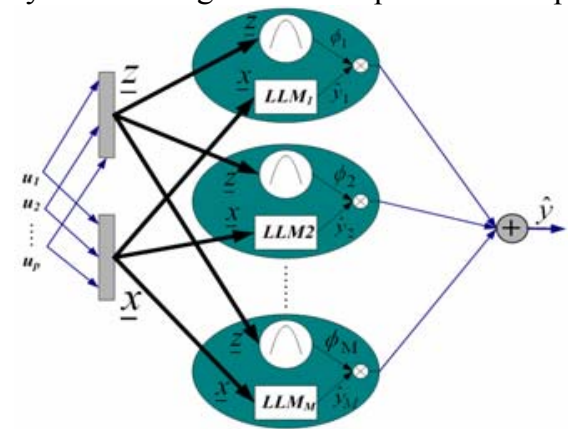

Fig. 7 Network structure of a Local Linear Neuro-Fuzzy model

In case of locally linear identification, the most imperative concern is the number of neurons. It is desirable that the number of neurons be as small as possible. The LOLIMOT algorithm is started from one neuron and gradually continues to arrive the neuron that shows an acceptable error based on sum of squared error curve so that the suitable number of neuron is distinct during the identification. Below are the brief five basic steps to identify the cement rotary kiln model [11, 19]:

1) Start with one initial model of cement rotary kiln,

2) Find worst Locally Linear Model that has maximum local loss function.

3) Check all hyper-rectangles to split (through).

(3a) Construction of the multi-dimensional fuzzy membership functions for both hyper rectangles.

(3b) Construction of all validity functions.

(3c) Local estimation of the rule consequent parameters for both newly generated LLMs.

(3d) Calculation of the loss functions for the current overall model.

4) Find best division (the best of the alternatives checked in

Step 3, and increment the number of LLMs: $M \rightarrow M+1$ ).

5) Test for convergence.

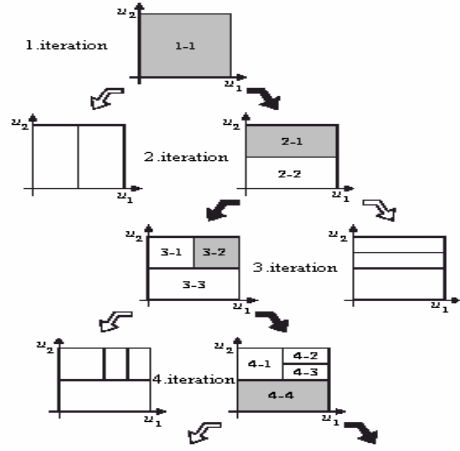

Fig. 8 Operation of the LOLIMOT algorithm in the first five Iterations for a two-dimensional input space [10]

As shown in Fig. 9, the optimal number of neurons for the first LLNF network, which has back end temperature as output, is two. More neurons do not affect significant reduction of error. It shows that a LLNF with two neurons can model the plant adequately.

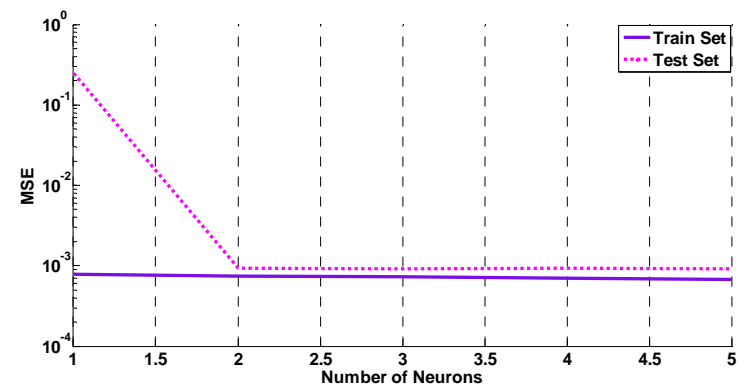

Fig. 9 Error on train and test data respect to different number of neurons

\section{Determining Inputs for Network}

One of the important factors in network operation is to select proper inputs; if the inputs do not have enough information and dynamism, identification process will fail. In order to determine the proper inputs of the network, it is necessary to know that each input has its own particular duration of effect on the output. This is a problem that we have during the identification process. With regards to the pre-knowledge about the kiln properties, the range of inputs dynamism is obtained then through trial and error during identification, the best number of input and output dynamics are obtained. The best numbers of dynamics used for identification are presented in table 4.

TABLE IV THE BEST NUMBER OF DYNAMICS FOR THE INPUTS AND OUTPU
\begin{tabular}{|c|c|c|c|c|}
\hline & \multicolumn{5}{|c|}{ Number of Dynamics } \\
\hline Variable & Ka & CO & BE & Pre \\
\hline Mat & 2 & 2 & 5 & 5 \\
\hline Fuel & 2 & 2 & 3 & 2 \\
\hline Ks & 2 & 2 & 2 & 3 \\
\hline Fan & 2 & 3 & 11 & 4 \\
\hline Ap & 2 & 4 & 3 & 2 \\
\hline Ka & 2 & - & - & - \\
\hline CO & - & 7 & - & - \\
\hline BE & - & - & 10 & - \\
\hline Pre & - & - & - & 11 \\
\hline
\end{tabular}




\section{E.} kiln

In reference to the previous sections and the contribution from the final process data, eight Nonlinear LLNF models for four kiln outputs have been obtained. The first four models relate to prediction kiln outputs model. Figures 10 to 13 show model output and real output from MISO1 to MISO4 for test data with prediction identification. The second four relate to simulator kiln outputs model. Figures 14 to 17 show model output and real output from MISO1 to MISO4 for test data with simulation identification.

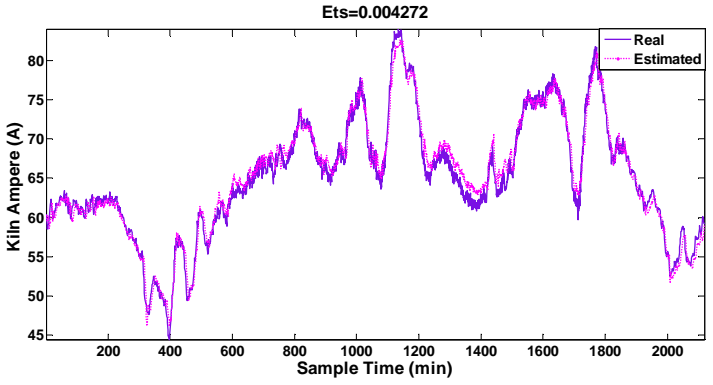

Fig. 10 Comparing between Prediction model and Real data for Kiln ampere output (5 min prediction horizon)

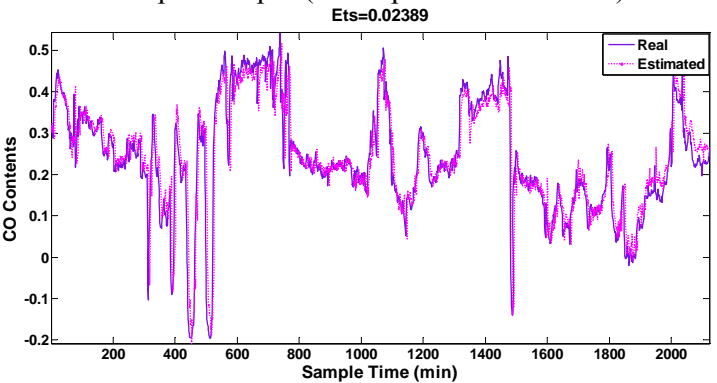

Fig. 11 Comparing between Prediction model and Real data for $\mathrm{CO}$ content output (5 min prediction horizon)

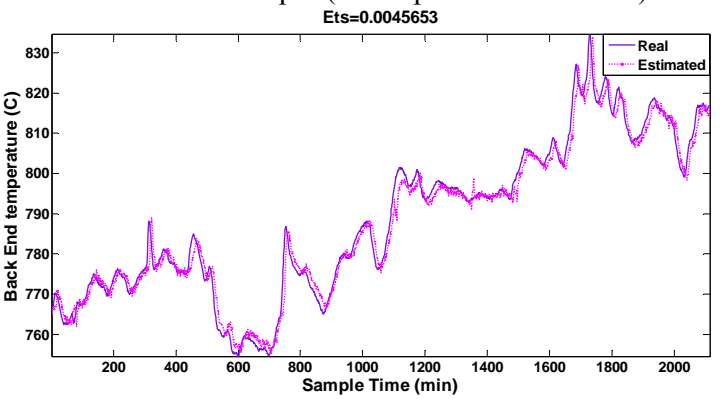

Fig. 12 Comparing between Prediction model and Real data for Back End temperature output (10 min prediction horizon)

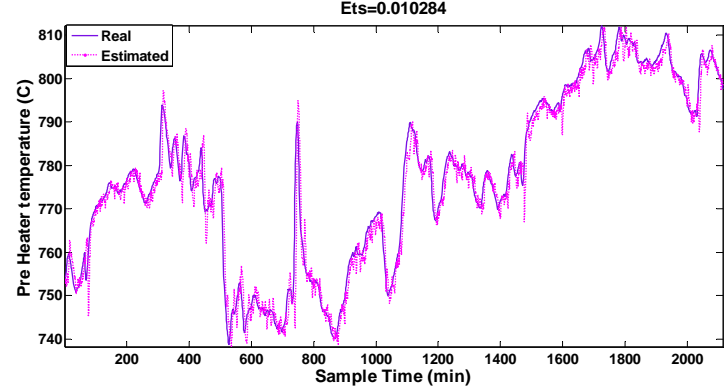

Fig. 13 Comparing between Prediction model and Real data for Pre-heater temperature output (10 min prediction horizon)

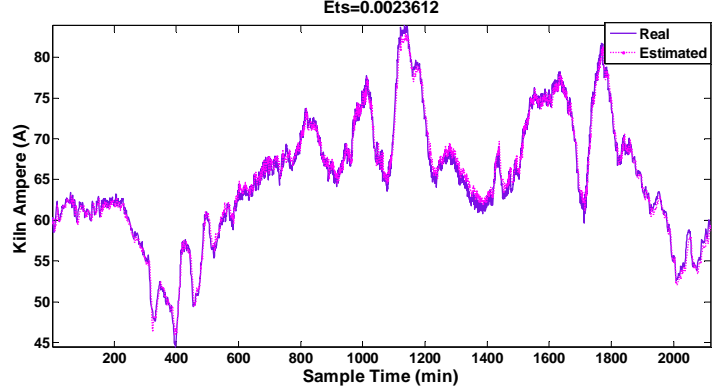

Fig. 14 Comparing between Simulation model and Real data for Kiln ampere output

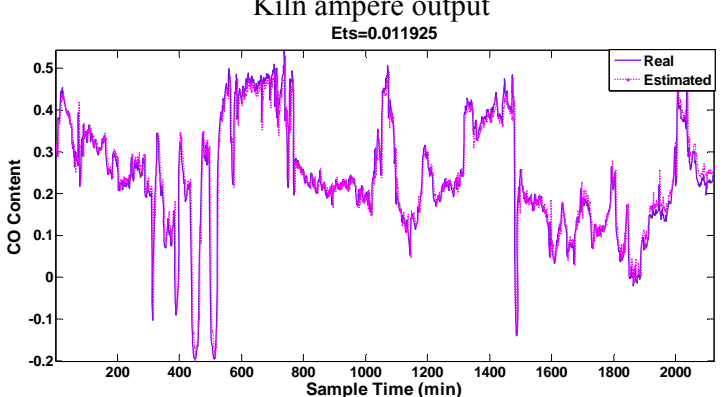

Fig. 15 Comparing between Simulation model and Real data for CO content output

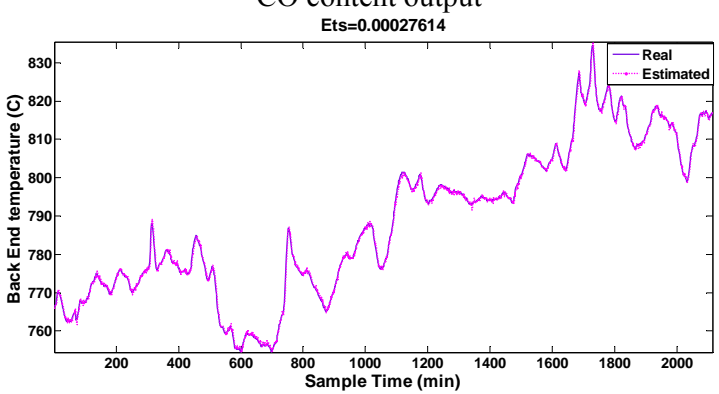

Fig. 16 Comparing between Simulation model and Real data for Back End temperature output Ets $=0.00050687$

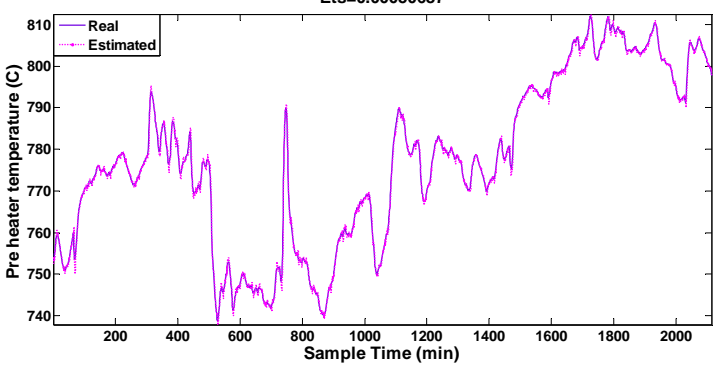

Fig. 17 Comparing between Simulation model and Real data for Pre heater temperature output

For different operation between two models we calculate root mean square error (RMSE) for test data which are shown in table 5 .

\begin{tabular}{|c|c|c|c|c|}
\hline Method & $\mathrm{Ka}$ & $\mathrm{CO}$ & $\mathrm{BE}$ & Pre \\
\hline Simulation & 0.00236 & 0.01192 & 0.00027 & 0.00050 \\
\hline 1 min Prediction & 0.00124 & 0.00222 & 0.00003 & 0.00003 \\
\hline 5 min Prediction & 0.00427 & 0.02389 & 0.00092 & 0.00190 \\
\hline 10 min Prediction & 0.00975 & 0.05161 & 0.00456 & 0.01028 \\
\hline 15 min Prediction & 0.01633 & 0.06930 & 0.00974 & 0.02229 \\
\hline
\end{tabular}


As you can see in table 5, the predictor model for one step prediction has less error than simulator model. However, as it was mentioned before, the simulator model could simulate kiln behavior after training without the need of real kiln output data, but only by applied inputs to this model. It is impossible to use prediction model for this purpose. This advantage of simulator model allows for utilizing the obtained locally linear model parameters for designing local controllers.

Here, we observe that an increase in prediction horizon increases the error in modeling. This increase of error for ten minutes prediction is between 2 to 7 percent. This means that with respect to the fastest time constant( three minutes in kiln) accompanied with the previously obtained predictor models; it is possible to predict the output for the fastest settling time with more accuracy within 10 percent of output that is acceptable rate for fault detection.

\section{CONCLUSION}

In this paper, identification of nonlinear predictor and simulator models by LLNF technique for cement rotary kiln is discussed and presented. In kiln identification, suitable sampling time, noise elimination, data normalization and inputs channels delay estimation are essential. In other words, without proper selection of parameters, identification will not be accurate. After the preprocessing on the data and obtaining a rich data set, two nonlinear predictor and simulator models for all the kiln outputs by LLNF and LOLIMOT algorithm are presented. The simulator model for cement rotary kiln is presented for the first time. Also, the predictor model with accuracy rate in fifteen minutes prediction horizon for all the kiln outputs is presented. The result shows that predictor model can be used for fault detection. Furthermore, the accuracy of simulator models can be applied in simulation and designing of model-based controllers.

\section{ACKNOWLEDGMENT}

The author and the co-author are grateful to Saveh Cement Company for their kind help and technical assistance during this project.

\section{REFERENCES}

[1] Devedzic, H., Knowledge-Based Control of Rotary Kiln, the proceedings of the International IEEE/IAS Conference on Industrial Automation and Control: Emerging Technologies, pp. 452 - 458, Taipei, 1995.

[2] Akalp, M., Dominguez, A. L. and Longchamp, R., Supervisory Fuzzy Control of a Rotary Cement Kiln, the Proceedings IEEE Electrotechnical Conference, vol. 2, pp. 754-757, 1994.

[3] Lu, J., Huang, L., Hu, Z., Wang, S., Simulation of gas-solid, two phase flow, coal combustion and raw meal calcination in a pre-calciner. ZKG International 57 (2), 55-63, 2004.

[4] Mastorakos, E., Massias, A., Tsakiroglou, C.D., Goussis, D.A. Burganos, V.N., CFD predictions for cement kiln including flame modeling, heat transfer and clinker chemistry. Applied Mathematical Modeling 23, 55-76, 1999

[5] Mujumdar, K.S., Ranade, V.V., Coupling of CFD models with different time scales - a case study of rotary cement kiln. In FLUENT Users Group Meeting held at Pune, 2003.

[6] Modigell, M.; Liebig D.; Munstermann, S. Calculation of the clinker burning process using thermochemical process simulation. ZKG Int., 55 (7), 38-46, 2002.
[7] Mujumdar, K.S., Ranade, V.V., Simulation of rotary cement kilns using a one-dimensional model. Transactions of IChemE, Part A, Chemical Engineering Research and Design 84 (A3), 165-177, 2006.

[8] T. Takagi, M. Sugeno, "Fuzzy identification of systems and its applications to modeling and control," IEEE Tran. Systems, Man and Cybernetics, vol. 15, pp. 116-132, 1985.

[9] J. R. Jang, "ANFIS: Adaptive network based fuzzy inference system," IEEE Tran. Systems, Man and Cybernetics, vol. 23, no. 3, , pp. 665-685, 1993.

[10] O. Nelles, Nonlinear system identification. Berlin: Springer Verlag, 2001.

[11] O. Nelles, "Local linear model tree for on-line identification of time variant nonlinear dynamic systems," International Conference on Artificial Neural Networks (ICANN), pp. 115-120, Bochum-Germany, 1996.

[12] Noshiravani, R., Identification of a Rotary Cement Kilns, Master of Science thesis (in Farsi), K. N. Toosi University of Technology, Tehran, Iran, 2005

[13] Iman Makaremi, Alireza Fatehi, Babak Nadjar Araabi, Morteza Azizi, Ahmad Cheloeian, "Identification and Abnormal Condition Detection of a Cement Rotary Kiln," 17th IFAC world congress, Seoul, Korea, July 6$11,2008$.

[14] Maryam Fallahpour, Alireza Fatehi, Babak Nadjar Araabi, Morteza Azizi“A neuro-fuzzy Controller for Rotory cement Kiln," the 17th World Congress The International Federation of Automatic ControlSeoul, Korea, July 6-11, 2008.

[15] I. Makaremi, "Intelligent Condition Monitoring of a Cement Rotary Kiln”, M.Sc. Thesis,KN Toosi Univ. of Tech, Feb 2007.

[16] Fallahpour, M., Fuzzy Controllers for Rotary Cement Kilns, Master of Science thesis (in Farsi), K. N. Toosi University of Technology, Tehran, Iran, 2007.

[17] Y.Zhu, Multivariable system identification for process control. Elsevier science Ltd, 2001.

[18] Iman Makaremi, Alireza Fatehi, Babak Nadjar Araabi, "Lipschitz Numbers: A Medium for Delay Estimation," 17th IFAC world congress, Seoul, Korea, July 6-11 2008.

[19] O. Nelles and R. Isermann, "Basis function networks for interpolation of local linear models," Proc. of IEEE Conference on Decision and Control, pp. 470-475, Kobe, Japan, 1996.

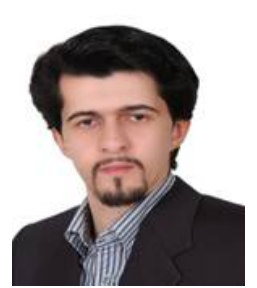

Masoud Sadeghian received the B.S degree in Electrical Engineering from Islamic Azad University of Najafabad in 2006, and the M.Sc. degree in Mechatronics Engineering at Sharif University of Technology International Campus, in 2009. His main interests are identification and modeling, fault diagnosis and prognosis, intelligent control and robotics.

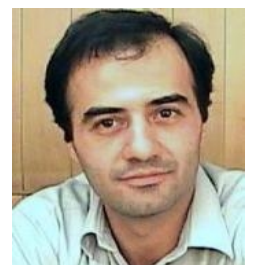

Alireza Fatehi received the M.Sc. degree from the University of Tehran, Iran, in 1995, and the Ph.D. degree from University of Tohoku, Japan, in 2001.He is an Assistant professor of K.N. Toosi University of Technology, Tehran, Iran, as well as director of Advanced Process Automation Control (APAC) and head of Mechatronics Engineering department of K.N. Toosi University of Technology. His research interests include intelligent control, multiple control, process control, and systems identification. 